University of Nebraska - Lincoln

DigitalCommons@University of Nebraska - Lincoln

$10-27-2006$

\title{
Electronic structure and polymerization of a self-assembled monolayer with multiple arene rings
}

\author{
D.Q. Feng \\ University of Nebraska-Lincoln, nkfdq@bigred.unl.edu \\ David Wisbey \\ University of Nebraska-Lincoln, bigwiz7@yahoo.com \\ Yaroslav B. Losovyj \\ Louisiana State University at Baton Rouge, ylozovyy@indiana.edu \\ Y. Tai
}

Angewandte Physikalische Chemie, Universität Heidelberg, Im Neuenheimer Feld 253, D-69120

Heidelberg, Germany

M. Zharnikov

Angewandte Physikalische Chemie, Universität Heidelberg, Im Neuenheimer Feld 253, D-69120

Heidelberg, Germany

See next page for additional authors

Follow this and additional works at: https://digitalcommons.unl.edu/physicsdowben

Part of the Physics Commons

Feng, D.Q.; Wisbey, David; Losovyj, Yaroslav B.; Tai, Y.; Zharnikov, M.; and Dowben, Peter A., "Electronic structure and polymerization of a self-assembled monolayer with multiple arene rings" (2006). Peter Dowben Publications. 108.

https://digitalcommons.unl.edu/physicsdowben/108

This Article is brought to you for free and open access by the Research Papers in Physics and Astronomy at DigitalCommons@University of Nebraska - Lincoln. It has been accepted for inclusion in Peter Dowben Publications by an authorized administrator of DigitalCommons@University of Nebraska - Lincoln. 


\section{Authors}

D.Q. Feng, David Wisbey, Yaroslav B. Losovyj, Y. Tai, M. Zharnikov, and Peter A. Dowben 


\title{
Electronic structure and polymerization of a self-assembled monolayer with multiple arene rings
}

\author{
D.-Q. Feng, ${ }^{1}$ D. Wisbey, ${ }^{1}$ Ya. B. Losovyj, ${ }^{2}$ Y. Tai, ${ }^{3}$ M. Zharnikov, ${ }^{3, *}$ and P. A. Dowben ${ }^{1, \dagger}$ \\ ${ }^{1}$ Department of Physics and Astronomy and the Nebraska Center for Materials and Nanoscience, University of Nebraska-Lincoln, \\ Lincoln, Nebraska 68588-0111, USA \\ ${ }^{2}$ Center for Advanced Microstructures and Devices, Louisiana State University, 6980 Jefferson Highway, \\ Baton Rouge, Louisiana 70806, USA \\ ${ }^{3}$ Angewandte Physikalische Chemie, Universität Heidelberg, Im Neuenheimer Feld 253, D-69120 Heidelberg, Germany
}

(Received 8 June 2006; revised manuscript received 4 August 2006; published 27 October 2006)

\begin{abstract}
We find evidence of intermolecular interactions for a self-assembled monolayer (SAM) formed from a large molecular adsorbate, $\left[1,1^{\prime} ; 4^{\prime}, 1^{\prime \prime}\right.$-terphenyl $]-4,4^{\prime \prime}$-dimethanethiol, from the dispersion of the molecular orbitals with changing wave vector $k$. With the formation self-assembled molecular (SAM) layer, the molecular orbitals hybridize to electronic bands, with indications of significant band dispersion of the unoccupied molecular orbitals. The electronic structure is also seen to be dependent upon temperature, and cross linking between the neighbor molecules, indicating that the electronic structure may be subtly altered by changes in molecular conformation and packing.
\end{abstract}

DOI: 10.1103/PhysRevB.74.165425

PACS number(s): 78.40.Me, 73.20.-r, 79.60.-i

\section{INTRODUCTION}

Most molecules are dielectrics, or can at least be considered as nominal insulators as long as the highest occupied molecular orbital (HOMO) to lowest unoccupied molecular orbital (LUMO) gap is larger than $3 k_{B} T$. Effectively, molecules have band gaps often centered about the Fermi level or chemical potential. Molecules also have molecular orbitals that are often extensively delocalized so the states are not restricted to a single atom (or even a localized group of atoms), but does increased molecular orbital delocalization lead to better or worse dielectric properties? Good molecular dielectrics, such as the molecular ferroelectrics, ${ }^{1}$ have significant intramolecular band structure, ${ }^{2}$ so molecular orbital delocalization, by itself, is not an indication of a conduction channel.

Self-assembled molecular (SAM) layers may be good insulators, ${ }^{3}$ but a number of practical problems must be faced before such layers are used as molecular dielectrics in the single molecule thickness limit. Among these obstacles to using SAM layers as a dielectric layer are the very significant problems associated with forming the second, or "upper" contact. Problems occur because the molecular tilt angles, generally adopted by adsorbed thiol molecules alkane or otherwise, are tilted off the surface normal leading to a "parting of hair" like effect between surface domains. This leads to electrical shorts across the SAM layer. ${ }^{4,5}$ Metals deposited on top of a SAM layer, at the SAM to vacuum interface, may penetrate into and through the molecular layer, even for a polyaromatic molecule such as $\left[1,1^{\prime} ; 4^{\prime}, 1^{\prime \prime}\right.$-terphenyl $]-4$, 4 "-dimethanethiol (TPDMT). ${ }^{6}$

There is still considerable promise that SAM layers can be used as good dielectric layers in practical devices. ${ }^{3}$ Among other approaches, electron beam irradiation leads to the cross linking or quasi (two-dimensional) polymerization of $\left[1,1^{\prime} ; 4^{\prime}, 1^{\prime \prime}\right.$-terphenyl]-4,4'-dimethanethiol (TPDMT), making the SAM layer a network of chemically linked TPDMT molecules. ${ }^{7-9}$ Cross-linked SAM layers provide an effective barrier for the penetration of a metal adsorbate. The cross linking makes possible the formation of the top electrode and the cross-linked SAM layers can then play the role of an insulating layer between this electrode and the bottom gold electrode. ${ }^{7-9}$ Such organic dielectric layers would not only provide potentially very well defined ultrathin dielectric barriers, but the barrier width can be adjustable by physical or chemical means. Among other implications, this provides the possibility that organic self-assembled monolayers may be used as ultrathin insulating layers in electronic and spintronic devices, ${ }^{7-9}$ partly because such a layer might be more robust against pin-hole formation.

Electron and x-ray-induced modification of selfassembled monolayers has been studied for both aliphatic $^{10-13}$ and aromatic thiol-derived SAMs. ${ }^{13-19}$ Such physical treatment leads to a quasipolymerization in aromatic thiol-derived SAMs and changes in electronic structure, ${ }^{19}$ which we explore here in more detail for $\left[1,1^{\prime} ; 4^{\prime}\right.$, 1 "-terphenyl]-4,4"-dimethanethiol (TPDMT). At issue is how the changes from an extra-molecular band structure to an intramolecular band structure (of the related quasipolymer) affect the dielectric properties.

Extra- or inter-molecular band structure in molecular monolayers, resulting from the hybridization of molecular orbitals of adjacent molecules, has a long and very rich history spanning nearly three decades of surface science. ${ }^{20-24}$ For the films comprising of larger molecules, intramolecular band dispersion is far more commonly observed than extramolecular band dispersion. ${ }^{24-29}$ The extra-molecular electronic dispersion (band structure) in ordered assemblies of large molecules has been difficult to obtain experimentally because of the very small effective Brillouin zone (requiring exceptionally good wave vector resolution) and the generally weak intermolecular interactions, with only a few exceptions as in Refs. 24 and 26-34. On the other hand, intramolecular band dispersion in oligophenyl chain molecules is very much expected ${ }^{35,36}$ and commonly observed. ${ }^{36}$ Strong intermolecular interactions in well ordered aromatic self-assembled monolayers (SAMs) may give rise to significant hybridization of molecular orbitals, leading to intermolecular band formation, sufficient for observing the band dispersion in 
aromatic thiol-derived layers, ${ }^{28,37}$ as well as $\left[1,1^{\prime} ; 4^{\prime}\right.$, 1 "-terphenyl]-4,4"-dimethanethiol (TPDMT), as we show in this paper. Nonetheless, sample preparation and molecular conformation play a role in determining the details of electronic structure.

\section{EXPERIMENT AND THEORY}

$\left[1,1^{\prime} ; 4^{\prime}, 1^{\prime \prime}\right.$-terphenyl $]-4,4^{\prime \prime}$-dimethanethiol

(TPDMT) forms densely packed and highly ordered self-assembled monolayers (SAMs) on $\mathrm{Au}(111)$ with an upright orientation of the terphenyl backbone somewhat (about $19.3^{\circ}$ ) canted off the surface normal and bound to the substrate via the thiolate linkage. ${ }^{6,18,38,39}$ The TPDMT SAMs studied here were prepared on evaporated $\mathrm{Au}(111)$ substrates, by what has become a standard immersion procedure. ${ }^{40}$ The exact details for TPDMT can be found elsewhere. ${ }^{7,38}$

The electronic structure of both occupied and unoccupied molecular orbitals of the adsorbed $\left[1,1^{\prime} ; 4^{\prime}, 1^{\prime \prime}\right.$-terphenyl $]-4$, 4 "-dimethanethiol layers on $\mathrm{Au}(111)$ surfaces was investigated through combined high resolution ultraviolet photoemission and inverse photoemission studies. In both types of photoemission measurements, the binding energies were referenced to the Fermi edge of both gold and tantalum, measured at $80 \mathrm{~K}$, in intimate contact with the sample surface. All of the electron spectroscopy experiments were performed under ultrahigh vacuum conditions.

The high resolution ultraviolet photoemission spectra (UPS) were taken using a helium lamp at $h v=21.2 \mathrm{eV}$ (He I) and a Scienta 200 hemispherical electron analyzer at the Center for Advanced Microstructures and Devices (CAMD) synchrotron radiation facility in Baton Rouge, Louisiana. The combined resolution in our high resolution ultraviolet photoemission experiments was better than $10 \mathrm{meV}$, as inferred from the widths of the $\operatorname{Ar} 3 p_{3 / 2}(15.8 \mathrm{eV})$ and $3 p_{1 / 2}$ $(15.9 \mathrm{eV})$ lines in photoionization spectra, as described in detail elsewhere. ${ }^{41}$ The band structure of occupied (valence band) molecular orbitals was measured using this high resolution angle-resolved ultraviolet photoemission spectroscopy (ARUPS) at a photon energy of $21.2 \mathrm{eV}$.

The inverse photoemission spectra were obtained by using variable incident energy electrons with a fixed energy U.V. detector (a Geiger-Müller detector). The instrumental linewidth is $\sim 400 \mathrm{meV}$, as described elsewhere. . $^{2,19,26,42,43}$

The light polarization dependent ultraviolet photoemission measurements were carried out using synchrotron light, dispersed by a $3 \mathrm{~m}$ toroidal grating monochromator, at CAMD, as described in detail elsewhere. ${ }^{19,26,29,43,44}$ The combined resolution of the hemispherical electron energy analyzer and monochromator varied between 0.10 and $0.25 \mathrm{eV}$, with an angular acceptance of $\pm 1^{\circ}$. Because of the highly plane polarized nature of the dispersed synchrotron light through the toroidal grating monochromator, the large light incidence angles resulted in $\mathbf{E}$ more parallel to the surface normal ( $p$-polarized light), while smaller light incidence angles resulted in $\mathbf{E}$ residing more in the plane of the surface $(s$-polarized light) in the geometry of our experiment. Several photon energies were used for the light polarization de- pendent photoemission, but for the results reported here, we used a photon energy of $80 \mathrm{eV}$, as noted.

The light polarization dependence of the photoemission signal, related to a particular feature in the spectra, is based on the photoemission selection rules and is closely related to the specific symmetry of involved molecular orbitals. ${ }^{20-24}$ The partial cross section for photoemission varies according to the orientation of the light vector potential $\mathbf{A}$ of the incident plane polarized light and the symmetry of the initial state $\psi_{i}$, assuming that the final state wave function $\psi_{f}$ (for electrons collected along the surface normal) is fully symmetric

$$
\left(\frac{d \sigma}{d \Omega}\right)_{P E S} \propto\left|\left\langle\Psi_{f}|\mathbf{A} \cdot \mathbf{p}+\mathbf{p} \cdot \mathbf{A}| \Psi_{i}\right\rangle\right|^{2} \delta\left(E_{f}-E_{i}-h v\right),
$$

where $\mathbf{A} \cdot \mathbf{p}$ is the effective dipole operator. For the photoemission results reported here, the photoelectrons were collected normal to the substrate surface, i.e., $k_{\|}=0$ or $\bar{\Gamma}$, to preserve the highest possible local point group symmetry. This means that application of Eq. (1) to the light polarization dependent photoemission from a molecule of $C_{2 v}$ point group symmetry, with the high symmetry axis along the surface normal, in molecular orbitals of $a_{1}$ symmetry becoming enhanced in $p$-polarized light whereas $b_{1}$ and $b_{2}$ symmetry molecular orbitals are enhanced with $\mathbf{E}$ residing more in the plane of the surface ( $s$-polarized light). Both light incidence angles as well as photoelectron emission angles, reported herein, are given with respect to the substrate surface normal.

Cross linking of aromatic SAM layers has been fairly well characterized, ${ }^{7-9,13-19}$ and we have adopted and modified those procedures to address some of the experimental constraints. The cleavage of phenyl $\mathrm{C}-\mathrm{H}$ bonds, during radiation induced cross linking, has been confirmed by the intensity decrease of the IR modes, ${ }^{6}$ while the stability of the aromatic skeleton of polyphenyl thiols leads to the very low level of the irradiation-induced thickness reduction. ${ }^{13}$ The evidence for the intermolecular cross linking is provided by the higher resistance of the irradiated areas to wet chemical etching ${ }^{6}$ and the demonstrated fabrication of freestanding nanosheets of cross-linked polyphenyl SAM layers from irradiated areas of $1,1^{\prime}$ biphenyl-4-thiol. ${ }^{45}$ The cross linking should be accompanied by a relative torsion rotation of the individual rings; otherwise, in view of the directionality of C-C bonds, a stable two-dimensional (2D) network cannot be produced, as noted later. In our experiments, the cross linking of the TPDMT films was undertaken in situ through exposure to $50 \mathrm{eV}$ electrons or to a broadband of synchrotron white (zero order) light through a 3 toroidal grating monochromator attached to a bending magnet at the CAMD. Electron beam exposures were estimated from the current density $\left(5 \mu \mathrm{A} / \mathrm{cm}^{2}\right)$ uniform illuminating the sample. The white light beam provides broadband soft $\mathrm{X}$-ray radiation $(20-160 \mathrm{eV})$ centered around $70 \mathrm{eV}$, and where the integrated power is approximately $0.1 \mathrm{~W}$ at $100 \mathrm{~mA}$ of the ring current. ${ }^{46-48}$ We find that nearly complete cross linking occurs by an exposure of 50 to $54 \mathrm{mC} / \mathrm{cm}^{2}$ of $50 \mathrm{eV}$ electrons, or 500-600 mAmin of synchrotron white light. ${ }^{19}$ The electron exposure threshold is consistent with an optimal dose of 
$40-45 \mathrm{mC} / \mathrm{cm}^{2}$ of $10 \mathrm{eV}$ electrons found in previous studies. ${ }^{7,8,19}$ A further exposure only leads to unnecessary degradation of the TPDMT film. ${ }^{7,18}$

The model calculations of the electronic structure of $[1$, $1^{\prime} ; 4^{\prime}, 1^{\prime \prime}$-terphenyl]-4,4"-dimethanethiol (TPDMT) and a pair of the separated and cross-linked TPDMT molecules were undertaken using PM3-NDO (neglect of differential overlap) with the HyperChem package, ${ }^{49,50}$ as has been undertaken successfully elsewhere. ${ }^{19,43,51,52}$ A calculated density of states was obtained by applying equal Gaussian envelopes of $1 \mathrm{eV}$ width to each molecular orbital at the ground state binding energies (to account for the solid state broadening in photoemission) and then summing, together with a rigid energy shift of a typical value of $4.7 \mathrm{eV}$ applied to the calculated electronic structure. . $^{, 19,23,24,26,35,43,51-56}$ The calculations do not account for photoemission and inverse photoemission matrix element effects. Furthermore, both photoemission and inverse photoemission are final state spectroscopies, so that comparison with this simple semiempirical theory, that includes neither excitations, multiconfigurational final states, matrix element effects nor finite temperature effects to ultraviolet photoemission (and inverse photoemission), must be considered as only qualitative.

The reasons for not exploiting the more "state-of-the-art" modern density functional (DFT) calculations are due to the failures of DFT to provide an accurate description of the electronic structure as determined by photoemission and inverse photoemission. ${ }^{57-60}$ DFT generally results in a HOMO-LUMO gap too small by as much as a factor of 2 or more. Consideration of the possible multiconfigurational final states ${ }^{60-62}$ is likely to provide a more accurate representation of the spectra, but orbital symmetry information is generally lost. A proper calculation of electronic structure should include the substrate (to properly account for charge transfer, thiolate formation, and charge screening), exchange and correlation energies, ${ }^{63}$ but such slab calculations, ${ }^{37,57,64}$ given the very large number of atoms per unit cell (39 atoms from one thiolate adsorbate alone), are computationally very expensive, even if at all possible. Although a ground state calculation, our simple molecular orbital calculations do provide an effective (but not ideal) compromise, and like similar semiempirical molecular orbitals calculations (PM3, MNDO, INDO, CNDO) have successfully recovered much of the detail observed in large molecular systems using photoemission and inverse photoemission, including reproducing, with reasonable agreement, the highest occupied molecular orbital (HOMO) to lowest unoccupied molecular orbital (LUMO) gap, ${ }^{19,35,43,51,52}$ which DFT does not. In spite of the severe limitations of these simple molecular orbital calculations, they can be compared, with some effect, to DFT calculations. ${ }^{65}$

\section{ELECTRONIC STRUCTURE OF $\left[1,1^{\prime} ; \mathbf{4}^{\prime}\right.$, 1"-TERPHENYL]-4,4"-DIMETHANETHIOL}

An indication of the combined density of states, resulting from the molecular orbital electronic structure, is obtained from the combination of photoemission (taken at a photon energy of $21.2 \mathrm{eV}$ ) and inverse photoemission for the $\left[1,1^{\prime}\right.$; 4',1"-terphenyl]-4,4"-dimethanethiol (TPDMT) film, as shown in Fig. 1. The experimental gap between the highest occupied (HOMO) and lowest unoccupied (LUMO) molecular orbitals for TPDMT films is of $6.8 \pm 0.2 \mathrm{eV}$. This experimental value is in surprisingly good agreement with the corresponding semiempirical model calculations that provide an estimate of $7.7 \mathrm{eV}$ (given the limitations of the model calculations).

In the ground state of TPDMT on Au, the energy distance from the LUMO to $E_{F}$ is $3.2 \mathrm{eV}$. This placement of the chemical potential within the large HOMO-LUMO gap, suggests that this monomolecular film could act as an effective dielectric barrier layer in field effect transistors and tunnel magnetoresistive devices, ${ }^{3,4}$ even in a monolayer film, if pin holes through TPDMT films can be eliminated, as suggested by model calculations of polyphenyl dithiolates. ${ }^{66-68}$

The placement of the LUMO and the HOMO with respect to the Fermi level, from photoemission and inverse photoemission, is about $1 \mathrm{eV}$ larger than the approximately $2 \mathrm{eV}$ suggested by molecular conductance measurements of terphenyl dithiol and related species. ${ }^{68}$ If the band dispersion of the unoccupied molecular orbitals is considered, as suggested by the measurements discussed below, the agreement between molecular conductance spectroscopy and combined photoemission and inverse photoemission is quite good. Molecular conductance spectroscopy suggests that many similar molecules are $p$-type insulators, ${ }^{68}$ while from combined photoemission and inverse photoemission, we find that 1 , $1^{\prime}$-biphenyl-4,4'-dimethanethiol (Ref. 26) and $\left[1,1^{\prime} ; 4^{\prime}\right.$, 1"-terphenyl]-4,4"-dimethanethiol (TPDMT) are slightly $n$ type, or given that the majority carrier has not been ascertained, the Fermi level is closer the lowest unoccupied state. These difference between the results from combined photoemission and inverse photoemission studies and molecular conductance spectroscopy have been observed before in the study of 1,1'-biphenyl-4,4'-dimethanethiol (Ref. 26). No definitive explanation of the differences can be provided from the data at hand and we are limited to speculation. It may be that the underlying metal substrate strongly influences tunneling from occupied states in molecular conductance spectroscopy, particularly if there are defects in the molecular overlayer, or that band dispersion of the unoccupied molecular orbitals plays a significant role in determining the Fermi level to LUMO gap, as just noted.

The elucidation of the electronic structure of $\left[1,1^{\prime} ; 4^{\prime}\right.$, 1 "-terphenyl]-4,4"-dimethanethiol is aided by comparison of the experimental data with the occupied and unoccupied molecular orbitals derived from the semiempirical ground state calculations. A number of the molecular orbitals of TPDMT can be identified in both photoemission and inverse photoemission spectra, as shown in Fig. 1, where only some of the orbitals are shown, and Fig. 2, where all the orbitals are listed. The features (Fig. 1) that can be assigned to groups of molecular orbitals occur at the binding energies, $\left(E-E_{F}\right)$ relative to the Fermi level, of $-3.6 \pm 0.1 \mathrm{eV},-5.0+0.2 \mathrm{eV}$, $-6.4 \pm 0.1 \mathrm{eV},-7.9 \pm 0.2 \mathrm{eV}, \quad-8.7 \pm 0.1 \mathrm{eV},-9.9 \pm 0.2 \mathrm{eV}$ in the photoemission and $3.2 \pm 0.1 \mathrm{eV}, 4.5 \pm 0.2 \mathrm{eV}$ in inverse photoemission spectra (as indicated in Fig. 2). 


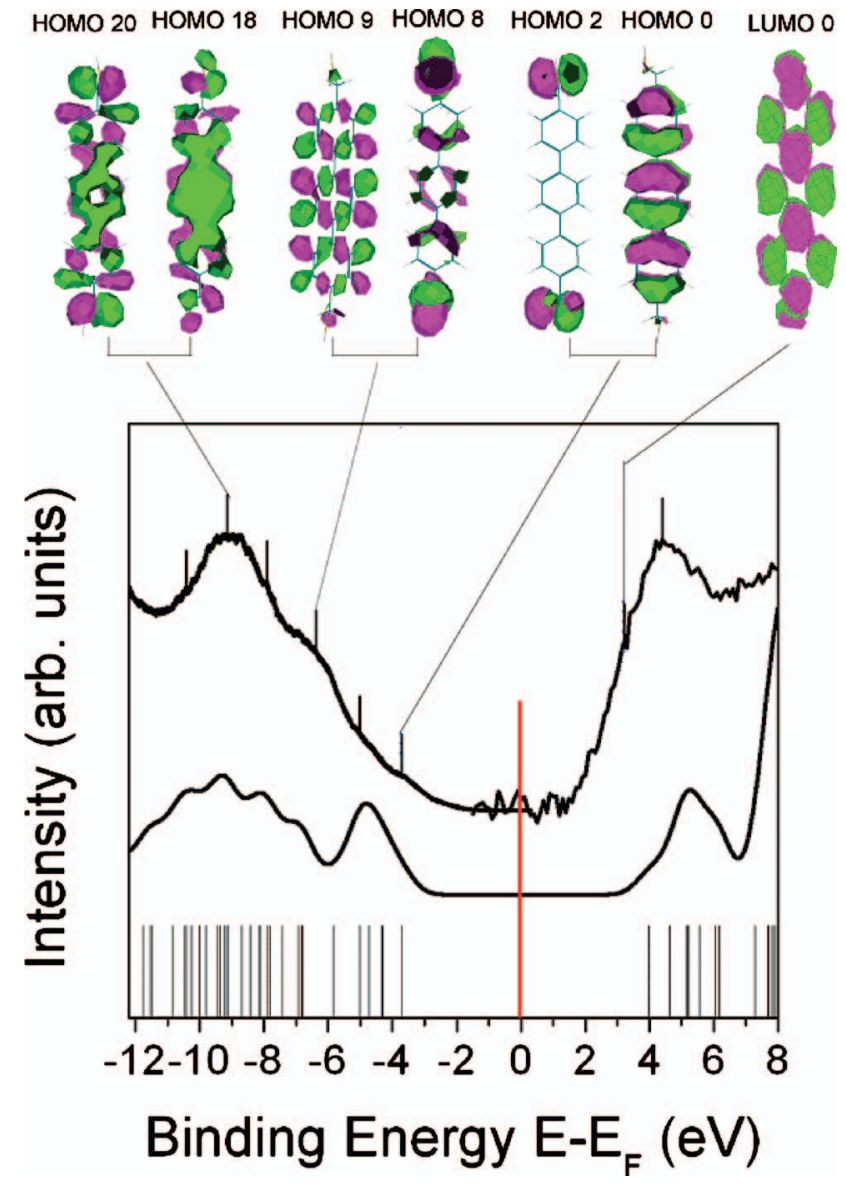

FIG. 1. (Color) Combined UPS (left) and IPES (right) spectra (top) of TPDMT/Au (top curve), along with the calculated ground state molecular orbital energies for a single TPDMT molecule (bottom line). The UPS and IPES signals are related to the contributions from the occupied and unoccupied molecular orbitals, respectively. Selected calculated molecular orbitals of TPDMT are shown on the top. The photoemission spectrum was taken with unpolarized He I $(21.2 \mathrm{eV})$, with the photoelectrons collected normal to the surface. The inverse photoemission spectrum was taken at normal incidence.

\section{INTERMOLECULAR BAND STRUCTURE}

In spite of the considerable off normal cant exhibited by TPDMT, 6,18,38,39 strong ordering within the molecular overlayer is evident in the band dispersion data. The high resolution angle dependent photoemission can be exploited to determine the dispersion (change in binding energy) as function of the wave vector parallel to the surface, $k_{\|}$, i.e., a strong dependence on the incidence angle for inverse photoemission or the emission angle for photoemission for molecules oriented along the surface normal. The value of $k_{\|}$is determined by

$$
k_{\|}=\sqrt{\frac{2 m}{\hbar} E_{k i n}} \sin (\theta)=0.512 \sqrt{E_{k i n} / \mathrm{eV}} \sin (\theta) \AA^{-1},
$$

where $E_{k i n}$ is the photoelectron kinetic energy and $\theta$ is the emission angle in the case of photoemission, ${ }^{20-24,28,29}$ or the incidence angle in the case of inverse photoemission. $2,23,24$

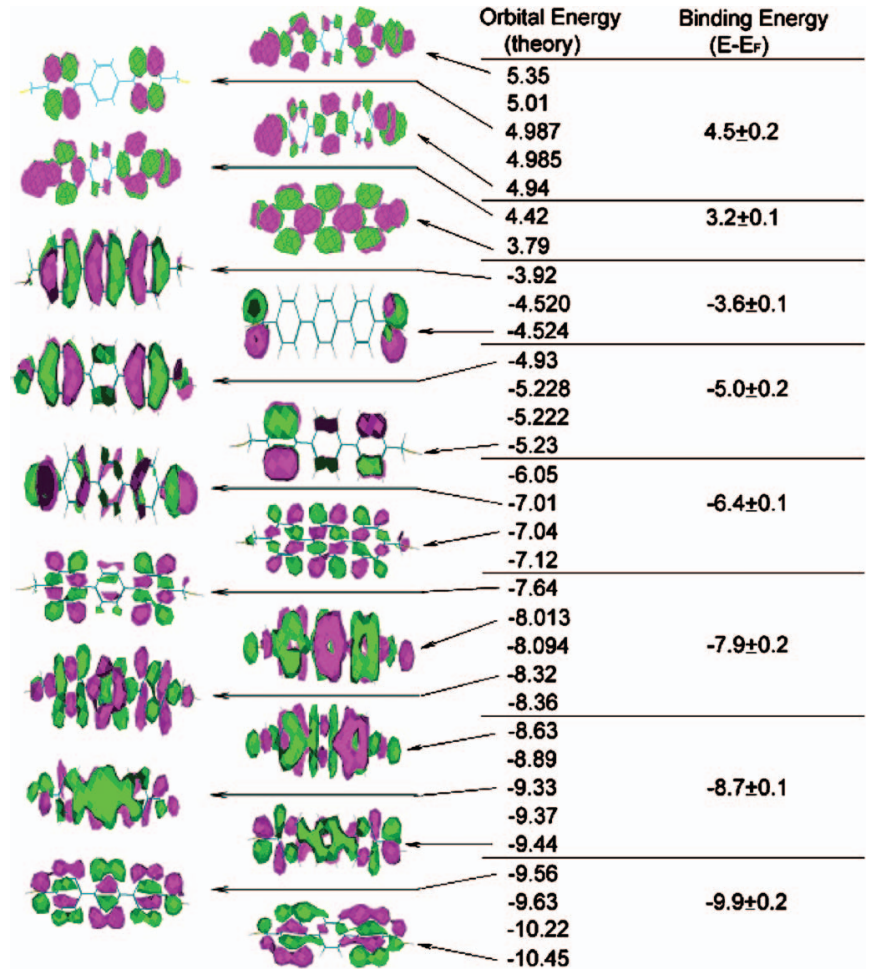

FIG. 2. (Color) The geometry optimization calculated ground state molecular orbital energies of TPDMT on Au substrate are compared with experimental binding energies with respect to the Fermi level. This includes a few unoccupied molecular orbitals down to the lowest unoccupied molecular orbital, as well as the occupied molecular orbitals referenced to the vacuum level. Selected molecular orbitals are shown left. The calculated ground state free molecular HOMO-LUMO gap is $7.7 \mathrm{eV}$.

There is evidence of dispersion of TPDMT molecular orbitals. From the band dispersion of the strongly sulfur weighted photoemission feature, at roughly 4 to $4.5 \mathrm{eV}$ binding energy, several band structure critical point are observed (denoted as $\bar{\Gamma}$ and the SBZE in Fig. 3). This periodicity of the band dispersion within the plane of the films is summarized in Fig. 3, where the peak position of the strongly sulfur weighted photoemission feature, following a detailed fitting of the experimental data, obtained at a photon energy of $21.2 \mathrm{eV}$, is plotted against the value of the $k_{\|}$vector along from $\bar{\Gamma}$ to the surface Brillouin zone edge (SBZE).

This occupied molecular orbital band dispersion of about 300 to $400 \mathrm{meV}$ for this sulfur weighted level is about what we would expect from the occupied bands in the calculated band structure of phenylthiolate SAM layers, ${ }^{37}$ and the 190 to $240 \mathrm{meV}$ band dispersion observed for the highest occupied molecular orbital of aromatic pentancene (Ref. 31) and thione 2-mercatobenzoxazole (Ref. 28) molecular layers. The only similar extra molecular band structure, for diphenyldimethyl dithiol, exhibited a similar value for the band dispersion and the band dispersion and suggests an intermolecular spacing of $5.0 \pm 0.1 \AA$ in the benzene planar direction. ${ }^{26}$

For dispersion along what is presumed to be the $\bar{\Sigma}$ line of the surface hexagonal Brillouin zone, the adjacent zone center occurs at about $1.25 \AA^{-1}$ (Fig. 3). Using the wave vector 


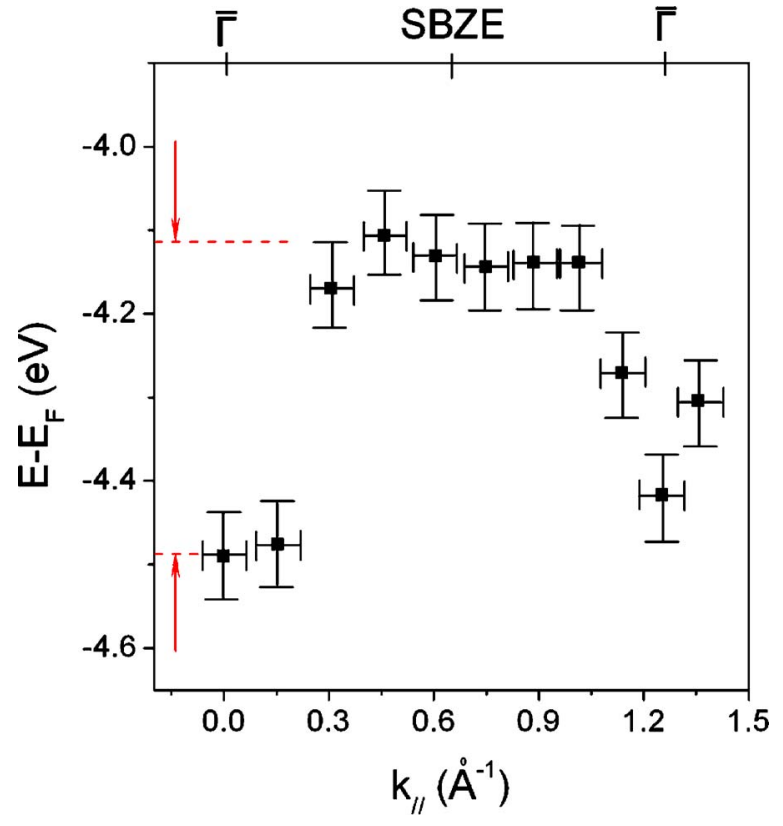

FIG. 3. (Color online) Intermolecular dispersion of a band originating from the sulfur weighted $\pi$ molecular orbitals of $\left[1,1^{\prime} ; 4^{\prime}\right.$, 1"-terphenyl]-4,4"-dimethanethiol (TPDMT) molecules assembled on $\mathrm{Au}(111)$ in the reduced zone scheme, determined from high resolution photoemission spectra taken with unpolarized $\mathrm{He}$ I $(21.2 \mathrm{eV})$ light.

difference between the center $(\bar{\Gamma})$ and edge $(\bar{M})$ of the Brillouin zone, we can estimate the intermolecular layer spacing in the TPDMT monolayer to be about $5.8 \pm 0.3 \AA$ from photoemission (Fig. 3). Unfortunately, multiple domains are sampled leading to a more cylindrical average in $k_{\|}$. This is evident in that the second Brillouin zone does not exhibit as quite as large a binding energy for the sulfur weighted band as is observed at $k_{\|}=0$, so this estimate of the lattice constant contains significant error weighted in the direction of larger lattice constants. Still this lattice constant is consistent with a $2 \times 2$ hexagonal structure. If only the sulfur is considered, this band dispersion provides a lattice constant that is close to one of lattice spacing of the herringbone structure proposed for terphenylmethylthiol and related molecules, ${ }^{69-71}$ although not the other. The rectangular lattice, such as is observed with 4'methylbiphenyl-4-yl-ethyl thiol and 4 ' methylbiphenyl-4yl-buthyl thiol, ${ }^{70,71}$ is more of a hexagonal packing consistent with a $6 \AA$ spacing, if super periodicities are NOT considered. These latter packing arrangements are very consistent with the photoemission derived band dispersion, if the band structure is from the TPDMT film and not from the substrate.

In some aromatic thiol SAM overlayers, ${ }^{69-71}$ with very high molecular coverages, a lattice constant of $5 \AA$ is reported and this can be reconciled with the critical points of our observed band dispersion under some conditions. A reciprocal lattice vector of $1.25 \AA^{-1}$, if the adlayer lattice is a rectangular lattice, would provide a $5.1 \pm 0.2 \AA$ lattice spacing in one direction. A roughly cylindrical average of such a rectangular lattice alone, resulting from multiple domains, is however difficult to reconcile with the experimental band dispersion data. Contributions from rectangular domains that contain a $5 \AA$ A lattice spacing, in combination with hexagonal domains, is possible but unlikely given the fact that such a combination would lead to a diminution of the observed band dispersion. Considering that any band structure we measure would sample several adlayer packing domains, the determination of a $5.8 \pm 0.3 \AA$ lattice constant, from band dispersion obtained from angle-resolved photoemission assuming an hexagonal packing arrangement, is very reasonable. The fact that the second Brillouin zone point is not well defined as $\bar{\Gamma}$, in the band structure, is certainly consistent with data obtained from superposition of several TPDMT domains of different orientations. Combinations of $5 \AA$ and 6 A lattice spacing with a roughly hexagonal packing have been seen in the LEED and molecular resolution STM results from some aromatic SAM layers, ${ }^{53-55,69-71}$ and cannot be excluded by our data either.

There is a tendency for thiol layers to adopt a $\sqrt{3}$ $\times \sqrt{3} R 30^{\circ}$ structure on $\mathrm{Au}(111)$, with an intermolecular spacing of about $5 \AA$. For SAMs formed from $\left[1,1^{\prime} ; 4^{\prime}\right.$, $1^{\prime \prime}$-terphenyl]-4-methanethiol, a commensurate $\sqrt{3} \times 2 \sqrt{3}$ structure, with an intermolecular spacing of about $5 \AA$, was observed by LEED and molecular resolution $\mathrm{STM},{ }^{69}$ while a $\sqrt{3} \times \sqrt{3} R 30^{\circ}$, with an intermolecular spacing again of about $5 \AA$, has been observed in other molecular resolution STM studies. ${ }^{72,73}$ The band structure critical points might be reconciled to such a $\sqrt{3} \times \sqrt{3} R 30^{\circ}$ hexagonal packing, if the band mapping is taken along the $\bar{T}$ line of the surface hexagonal Brillouin zone, toward the Brillouin zone edge $(\bar{K})$, but the second Brillouin center should not be observed in this case, so this structure remains difficult to reconcile with our data.

The strong sulfur weight of the occupied band (Fig. 3) does not provide a completely compelling case for intermolecular band dispersion, as this band dispersion could be dominated by either the more strongly chemisorbed sulfur at the gold interface or hydrogen terminated sulfur at the vacuum interface. More compelling evidence for intermolecular hybridization of molecular orbitals comes from the changes in the lowest unoccupied arene $\pi$ molecular orbital with changing wave vector (as indicated in Fig. 4). Band dispersion is evident from a comparison of the inverse photoemission spectra at two different wave vectors: zone center and for $k_{\|}=0.8 \AA^{-1}$. The binding energy shifts in the lowest unoccupied molecular orbitals suggests an unoccupied molecular orbital band dispersion of the order of $1 \mathrm{eV}$, with the changing of wave vector parallel to the surface. The band dispersion amplitude of $0.95 \mathrm{eV}$ or more, of the LUMO state, is much bigger than that of the occupied states $(0.4 \mathrm{eV})$, as a result of deeper potential of the occupied $\pi$ molecular orbitals of TPDMT. This is consistent with the expectations of the calculated band structure of phenylthiolate SAM layers. ${ }^{37}$ The nearly $1 \mathrm{eV}$ dispersion for the lowest unoccupied molecular orbitals places the LUMO closer to the Fermi level, with a separation of about $2 \mathrm{eV}$ (Fig. 4). This value is in reasonably good agreement with molecular conductance spectroscopy of similar molecules, ${ }^{68}$ as noted above. The complication of the molecular tilt angle and the interplay between the film structure, molecular conforma- 


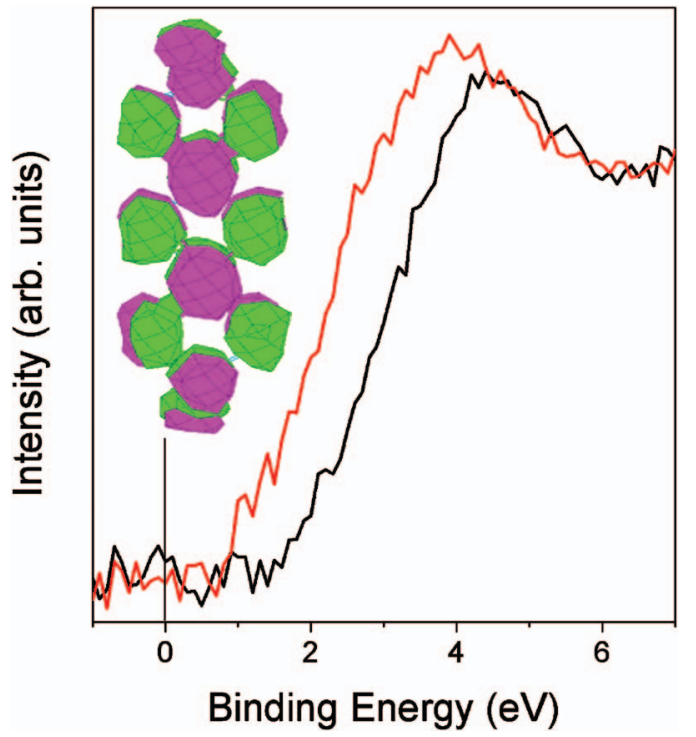

FIG. 4. (Color) Indications of intermolecular band dispersion from the LUMO $\pi$ molecular orbitals of $\left[1,1^{\prime} ; 4^{\prime}, 1^{\prime \prime}\right.$-terphenyl]-4, $4^{\prime \prime}$-dimethanethiol. The angle-resolved inverse photoemission spectra are shown for zone center (black) and for $k_{\|}=0.8 \AA^{-1}$ (red). The corresponding LUMO is shown in the margin.

tion, and attendant band structures does, however, need to be explored more systematically. This has only been indirectly suggested by prior studies. ${ }^{70,71}$

\section{COMPARISON OF THE ELECTRONIC STRUCTURE BETWEEN PRISTINE AND CROSS-LINKED [1,1'; $4^{\prime}, 1^{\prime \prime}$-TERPHENYL]-4,4"-DIMETHANETHIOL SAMS}

There are three major irradiation-induced processes: molecular decomposition, desorption of the film fragments, and cross linking of neighboring SAM constituents. ${ }^{13}$ While the decomposition and desorption processes prevail in aliphatic SAMs, cross linking is the dominant effect in the aromatic molecular layers, resulting in a quasipolymerization of the monomolecular film. ${ }^{13-19}$ The schematic of cross-linked TPDMT is shown in Fig. 5.

From the comparison of the combined photoemission and inverse photoemission derived highest occupied (HOMO) to lowest unoccupied (LUMO) molecular orbital gaps for the pristine and extensively irradiated TPDMT SAMs (Fig. 6), it is evident that cross linking leads to a reduction in the gap. The experimental HOMO-LUMO gaps are $6.8 \pm 0.2 \mathrm{eV}$ for the pristine [Fig. 6(a)] and $5.9 \pm 0.2 \mathrm{eV}$ for strongly irradiated [Fig. 6(b)] TPDMT films, in good agreement with our expectation from simple model calculations. The corresponding HOMO-LUMO gaps for the pristine and cross-linked TPDMT films from semiempirical model calculations are $7.6 \mathrm{eV}$ and $5.6 \mathrm{eV}$ respectively, as seen from Fig. 6. [Note that the latter cross-linked TPDMT films were exposed to $54 \mathrm{mC} / \mathrm{cm}^{2}$ of $50 \mathrm{eV}$ electrons (IPES) or white light, about $600 \mathrm{~mA} \cdot \min (\mathrm{UPS})$ in our experiments, for optimal crosslinking, as described in the experimental section.]

As seen in Fig. 6, the extensive cross linking does not alter the occupied molecular orbital binding energies (photo-

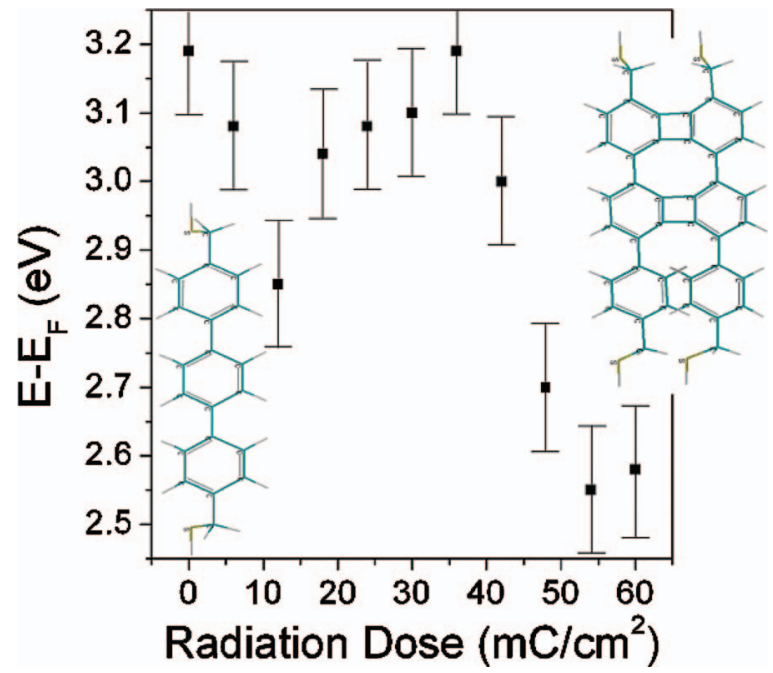

FIG. 5. (Color) The relative (with respect to the Fermi level) position of LUMO in the TPDMT SAM, obtained from the IPES data, as a function of irradiation dose (50 eV electrons). The insets show $\left[1,1^{\prime} ; 4^{\prime}, 1^{\prime \prime}\right.$-terphenyl]-4,4"-dimethanethiol (TPDMT) before (left) and after (right) electron-induced cross linking. The error bars indicate the relative error between the measurements and do not include the systemic errors arising from the instrumental function.

emission, on the left in Fig. 6) as significantly as those for the unoccupied molecular orbitals (inverse photoemission on the right in Fig. 6). This is illustrated in Fig. 5, where we plotted $E_{\mathrm{LUMO}}-E_{F}$, derived from the IPES data, as a function of the radiation dose. Recall that it is the lowest unoccupied molecular orbitals that provide evidence of strong intermolecular band dispersion (Fig. 4), so the cleavage of the phenyl $\mathrm{C}-\mathrm{H}$ bonds, during radiation induced cross linking, ${ }^{6}$ and the concomitant intermolecular cross linking should have a profound effect on the lowest unoccupied molecular orbitals.

As seen in Fig. 5, the influence of the irradiation leading to cross linking of the TPDMT film on the energy position of the unoccupied (right) molecular orbitals is highly nonlinear. There is an increase of the LUMO binding energy (a decrease in the value of $\left.E_{\mathrm{LUMO}}-E_{F}\right)$ with initial irradiation (around $10 \mathrm{mC} / \mathrm{cm}^{2}$ of $50 \mathrm{eV}$ electrons), and the position of the LUMO remains approximately constant up to a dose of $40 \mathrm{mC} / \mathrm{cm}^{2}$, which follows by a significant decrease in the value of $E_{\mathrm{LUMO}}-E_{F}$ with further irradiation.

Although the HOMO-LUMO gap is smaller for the strongly cross-linked (irradiated) TPDMT films (Fig. 6), such films are more dielectric than the pristine TPDMT films, as noted elsewhere. ${ }^{9,19}$ The increase in dielectric character with progressive cross linking (at least up to a point, before degradation leads to a decrease in dielectric properties) occurs in spite of the decreases in the HOMO-LUMO gap upon irradiation of the TPDMT films. This may be associated with the conjugation of the molecular orbitals along the oligophenyl backbone and molecular rigidity. In particular, irradiationinduced twisting of the individual rings along the terphenyl backbone can be of importance, ${ }^{19}$ and suggested by model studies of biphenyl dithiols. ${ }^{66}$ The only way to build a crosslinking network is the torsion of the individual rings in the $\mathrm{SAM}$ constituents, to make direct $\mathrm{C}-\mathrm{C}$ bonds to the neighbor 


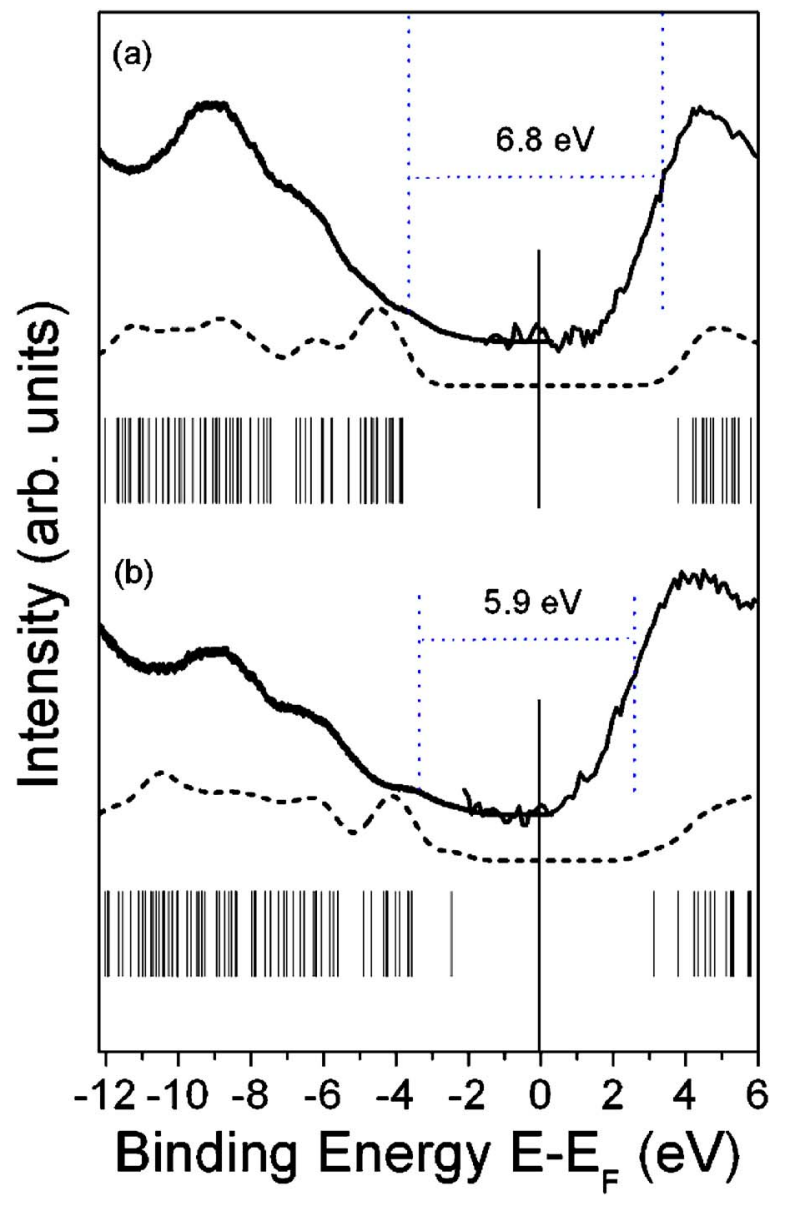

FIG. 6. (Color online) Combined UPS (left) and IPES (right) spectra (solid line) of pristine (a) and irradiated (b) TPDMT/Au, along with the calculated density-of-states (dashed line) for a pair of the separate and cross-linked TPDMT molecules. The UPS and IPES signals are related to the contributions from the occupied and unoccupied molecular orbitals, respectively. The experimental HOMO-LUMO gaps are indicated. The irradiated films were exposed to $54 \mathrm{mC} / \mathrm{cm}^{2}$ of $50 \mathrm{eV}$ electrons (IPES) and synchrotron white light exposure of about $600 \mathrm{~mA}$ min (UPS). The photoemission spectra were taken with unpolarized He I $(21.2 \mathrm{eV})$ light.

molecule. More detailed band structure measurements are surely indicated, as direct molecule-molecule interactions should play a role, even when the number of polyphenyl molecules between two electrodes is limited. ${ }^{74,75}$

\section{POSSIBLE CHANGING ORIENTATION AND CONFIGURATION}

Given that the terphenyl backbone is somewhat canted off the surface normal (about 19.3 $\left.{ }^{\circ}\right), 6,18,38,39$ while bound to the substrate via the thiolate linkage, profound light polarization dependence is not observed in the photoemission, as indicated in Fig. 3. The very small light polarization dependence of the valence band spectra with temperature (Fig. 7) can be understood on the basis of the partly preserved molecular orbital symmetries, schematically shown in Figs. 1 and 2, and the largely upright bonding orientation. Exploiting the known largely "upright" orientation of the TPDMT molecules, the pronounced photoemission feature at $-3.6 \pm 0.1 \mathrm{eV}$ (Fig. 7) can be attributed to occupied molecular orbitals of largely sulfur $p_{x, y}$ or largely from the arene (benzene) occupied $\pi$ molecular orbitals. Such a feature could exhibit enhancement in more $p$-polarized light as seen in photoemission (Fig. 7). Similarly, the photoemission feature at $-6.4 \pm 0.1 \mathrm{eV}$ is also dominated by arene (benzene) occupied $\pi$ molecular orbitals, but with contribution from the methyl $\sigma$ bonds and sulfur $p_{z}$, consequently resulting in a photoemission which resembles a mixture of benzene orbitals of different symmetries $\left(a_{1}+b_{1}+b_{2}\right)$ and is, thus, less likely to exhibit light polarization dependence in photoemission regardless of the molecular orientation. Occupied molecular orbitals containing strong $b_{1}$ symmetry character of benzene with $\pi$ and $\sigma$ bond weight contribute to the photoemission feature at $-8.7 \pm 0.1 \mathrm{eV}$ (Fig. 7), and can exhibit some light polarization dependent photoemission effects.

There are small changes in the light polarization dependent photoemission from the photoemission feature at $-8.7 \pm 0.1 \mathrm{eV}$ (Fig. 7) with temperature. The feature at $8.7 \pm 0.1 \mathrm{eV}$ becomes slightly enhanced in $p$-polarized light at lower temperatures, but is weakly enhanced with more $s$-polarized light at higher temperatures. Consistent with the temperature dependent photoemission intensities that deviate from expected Debye-Waller scattering behavior, ${ }^{76}$ these small changes in the light polarization dependent photoemission spectra strongly suggest that the orientation and conformation of TPDMT film changes with temperature. Note that the observed temperature dependent changes are fully reversible but the photoemission dichroism effects are far too small to claim a molecular orientation of the long molecular axis in an orientation within $10^{\circ}$ to the surface normal, consistent with the $19.3^{\circ}$ cant angle determined from x-ray absorption spectroscopy experiments (based generally on core to bound excitations). 6,13,38,39

Along with the LUMO shift that accompanies the cross linking of TPDMT, as discussed above, we observed another prominent effect of the irradiation-a significant change in the orientation of the TPDMT SAM at a relatively small irradiation dose (around $10 \mathrm{mC} / \mathrm{cm}^{2}$ for $50 \mathrm{eV}$ electrons). This is illustrated by Fig. 8 where the UPS spectra acquired at different light polarizations are presented, using polarized light at a photon energy of $80 \mathrm{eV}$. The changes in these spectra at going from a large incidence angle of $70^{\circ}$ ( $p$-polarized light) to a smaller incidence angle of $45^{\circ}(s+p$ polarized light) are far more significant for the modestly irradiated films (irradiation doses of $100 \mathrm{~mA}$ min) than for the pristine one, especially at $T=135 \mathrm{~K}$. Taking into account that the observed dichroism (i.e., light polarization dependence) is a fingerprint of molecular orientation, we interpret this light polarization dependence as a tendency for the TPDMT/Au to adopt a more upright orientation (placing the molecular axis closer to the surface normal), particularly at $135 \mathrm{~K}$, compared to the pristine TPDMT film which is densely packed and highly ordered with an average tilt angle the terphenyl moieties of $19.3^{\circ} .{ }^{38}$ The tilt of molecular axes between an orientation along the surface normal and parallel with the substrate normal will minimize the enhancement of the different photoemission features for each light polarization even the film is well ordered. 


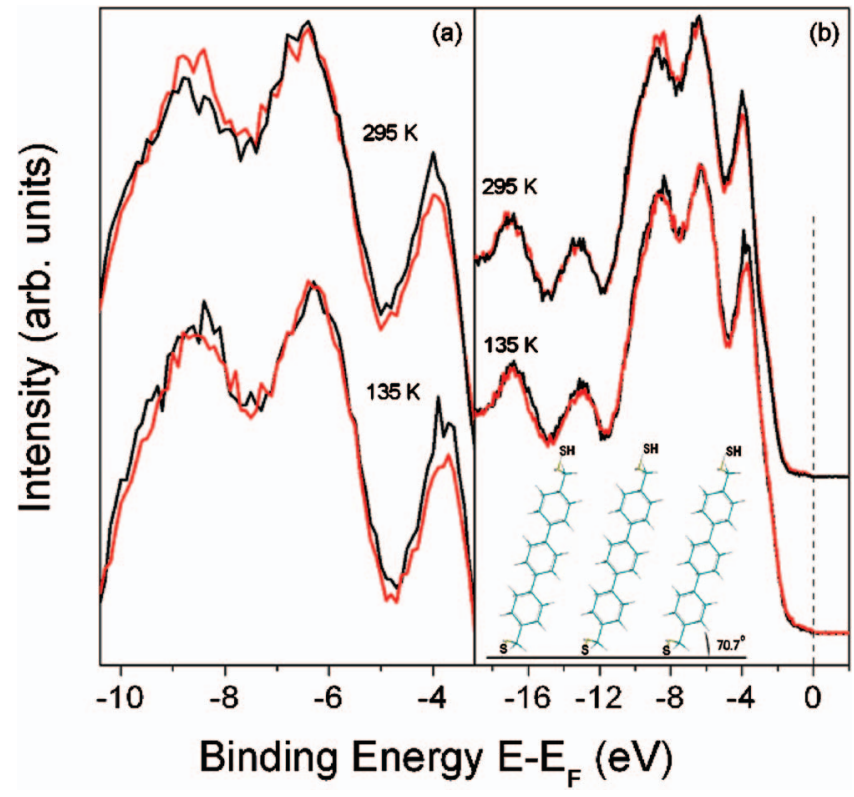

FIG. 7. (Color) Comparison of the light polarization photoemission spectra of TPDMT taken with $p$-polarized light (black lines) and $s+p$-polarized light (red lines) as a function of temperature. In (a), the energy region with the temperature reversible polarized light effects are enhanced compared to the broader energy scale in (b). The proposed model for the molecular arrangement in the TPDMT film at $295 \mathrm{~K}$, in accordance with Ref. 9, is shown in the inset. The spectra were taken at photon energy of $80 \mathrm{eV}$.

The shift of the LUMO towards the Fermi level at this level of cross linking (i.e., the exposure to $100 \mathrm{~mA}$ min of synchrotron white light or $10 \mathrm{mC} / \mathrm{cm}^{2}$ of $50 \mathrm{eV}$ electrons) may be associated with better molecular $\pi-\pi$ stacking. The capability of the TPDMT films to "improve" orientational order under physical treatment (say radiation induced partial dehydrogenation and cross linking) is consistent with the recent near edge $\mathrm{x}$-ray absorption fine structure (NEXAFS) spectroscopy studies which indicate a drastic decrease in the molecular tilt in TPDMT/Au upon the evaporation of a small amount of Ni. ${ }^{8}$ The evidence of temperature dependent light polarization effects for the differently irradiated films, as seen from Fig. 8, suggests that the film is more ordered at lower temperature, at least upon a moderate irradiation. Thus, the structure of TPDMT films appears to depend not only on the extent of cross linking but also on temperature.

The smaller light polarized enhancement for the extensively cross-linked TPDMT films, together with the small variations and changes in the relative intensities of the 3.9 and $8.8 \mathrm{eV}$ binding energy features in photoemission suggest that some further changes other than simply reorientation occur with irradiation, consistent with model calculations. The light polarization photoemission for the extensively irradiated film compared to the moderately irradiated ones indicates that the TPDMT SAM with nearly saturated cross linking has less ordering and the terphenyl moieties in adjacent molecules are not coplanar. With progressive irradiation doses much greater than $100 \mathrm{~mA}$ min. of synchrotron white light, the orientation ordering of the (increasingly cross linked) TPDMT film along the surface normal is partly lost

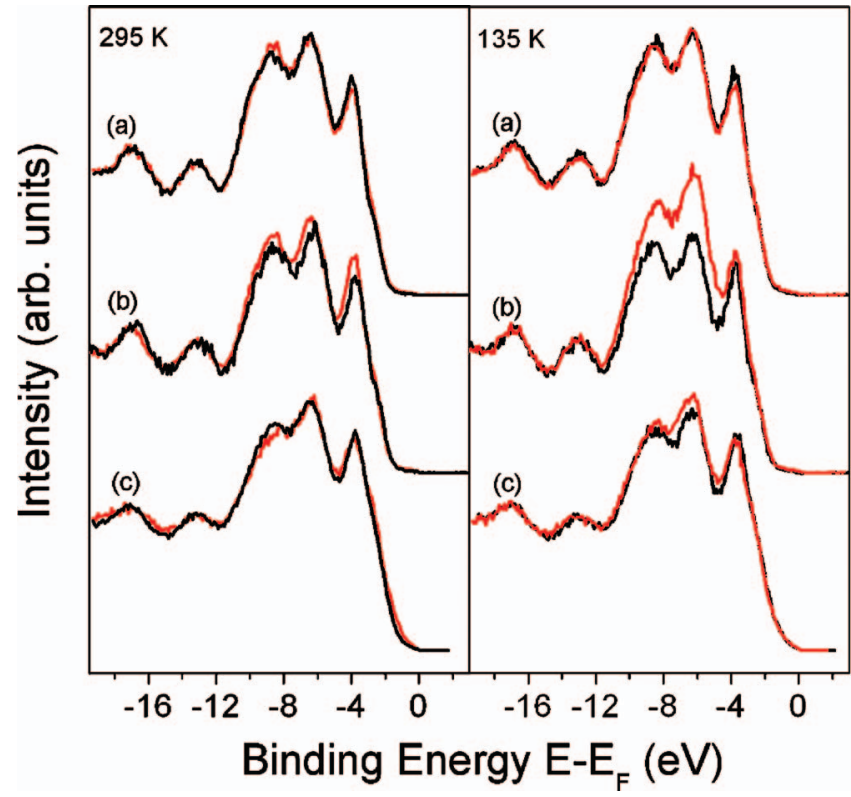

FIG. 8. (Color) Comparison of the photoemission spectra taken with linearly polarized $p$-polarized light (black) and $s+p$-polarized light (red) for the pristine (a) and irradiated (b,c) TPDMT film after its exposure to $100 \mathrm{~mA} \min (\mathrm{b})$ and $500 \mathrm{~mA} \min (\mathrm{c})$ of synchrotron white light. The data for $295 \mathrm{~K}$ (left panel) and $135 \mathrm{~K}$ (right panel) are shown. The spectra were taken at photon energy of $80 \mathrm{eV}$ and the photoelectrons collected at normal emission.

as indicated by loss of the significant light polarization dependence (particularly at $135 \mathrm{~K}$ ). The structural ordering is not lost completely, however, as has been clearly shown by NEXAFS measurements, ${ }^{17,18}$ but there is a combination of a molecular axis tilted away from the surface normal and a loss of coplanar benzene ring orientation, which leads to a significant decrease in light polarization effects in photoemission. This is consistent with a decrease in the coplanar orientation of adjacent molecular terphenyl backbones indicated by our model calculations.

\section{SUMMARY}

The TPDMT films, on gold substrates, are very well ordered molecular assemblies as illustrated in by the evidence of band dispersion (Fig. 3). Such in-plane ordering may be indicative of strong lateral interactions within the organic film, as has been suggested for $\left[1,1^{\prime} ; 4^{\prime}\right.$, 1"-terphenyl]-4-methanethiol (Ref. 72) phenylthiolate SAM layers, ${ }^{37}$ pentancene, ${ }^{31}$ thione 2-mercatobenzoxazole, ${ }^{28}$ diphenyldimethyl dithiol, ${ }^{26}$ and other thiol-derived SAMs. ${ }^{74,75}$ The strong intermolecular interaction is most likely responsible for the easily observed dispersion of the lowest unoccupied molecular orbitals. What is clearly indicated is that there is a crying need for further structural studies that address the molecular packing as well as registry with the metal substrate under a variety of conditions that include temperature and coverage. This has been indirectly suggested by prior studies ${ }^{70,71}$ but now there is a much 
clearer need to characterize SAM layers following different preparation techniques by scanning tunneling microscopy, low energy electron diffraction and the in-plane band structure mapping, to relate the resulting surface structures to molecular conformation, tilt angle, and packing density.

We found that the irradiated TPDMT films have a smaller HOMO-LUMO gap as compared to the pristine TPDMT films, which is consistent with theoretical calculation. The Fermi level placement, within the HOMO-LUMO gap suggests that strongly irradiated TPDMT films are more $n$-type insulating systems than the pristine TPDMT SAMs, and both molecular films have an electron structure consistent with a wide band gap insulator. The two dimensional quasipolymerization of TPDMT forms a dielectric barrier that can be better characterized than a polymerized benzene barrier layer. ${ }^{77}$

\section{ACKNOWLEDGMENTS}

This work was supported by the National Science Foundation through Grant No. CHE-0415421 and the NSF "QSPINS" MRSEC (DMR 0213808), the DFG (ZH 63 9/2), the German BMBF (05KS4VHA/4), the Center for Advanced Microstructures and Devices (CAMD) and the Louisiana Board of Regents. The authors would also like to acknowledge the support and assistance of M. Grunze.
*Corresponding author.

Electronic address: urz.uni-heidelberg.de

Corresponding author.

Electronic address: pdowben@unlinfo.unl.edu

${ }^{1}$ L. M. Blinov, V. M. Fridkin, S. P. Palto, A. V. Bune, P. A. Dowben, and S. Ducharme, Usp. Fiz. Nauk [Russian edition vol.] 170, 247 (2000); Phys. Usp. [English edition volume] 43, 243 (2000).

${ }^{2}$ Jie Xiao, L. G. Rosa, M. Poulsen, D.-Q. Feng, S. Reddy, J. M. Takacs, L. Cai, J. Zhang, S. Ducharme, and P. A. Dowben, J. Phys.: Condens. Matter 18, L155 (2006); J. Choi, P. A. Dowben, S. Ducharme, V. M. Fridkin, S. P. Palto, N. Petukhova, and S. G. Yudin, Phys. Lett. A 249, 505 (1998); Chun-gang Duan, W. N. Mei, J. R. Hardy, S. Ducharme, J. Choi, and P. A. Dowben, Europhys. Lett. 61, 81 (2003); J. Choi, C. N. Borca, P. A. Dowben, A. Bune, M. Poulsen, S. Pebley, S. Adenwalla, S. Ducharme, L. Robertson, V. M. Fridkin, S. P. Palto, N. Petukhova, and S. G. Yudin, Phys. Rev. B 61, 5760 (2000).

${ }^{3}$ M. L. Chabinyc, X. X. Chen, R. E. Holmlin, H. Jacobs, H. Skulason, C. D. Frisbie, V. Mujica, M. A. Ratner, M. A. Rampi, and G. M. Whitesides, J. Am. Chem. Soc. 124, 11730 (2002); E. Tran, M. Duati, V. Ferri, K. Mullen, M. Zharnikov, G. M. Whitesides, and M. A. Rampi, Adv. Mater. (Weinheim, Ger.) 18, 1323 (2006); D. Vuillaume, C. Boulas, J. Collet, J. V. Davidovits, and F. Rondelez, Appl. Phys. Lett. 69, 1646 (1996); C. Boulas, J. V. Davidovits, F. Rondelez, and D. Vuillaume, Microelectron. Eng. 28, 217 (1995); C. Boulas, J. V. Davidovits, F. Rondelez, and D. Vuillaume, Phys. Rev. Lett. 76, 4797 (1996).

${ }^{4}$ R. Haag, M. A. Rampi, R. E. Holmlin, and G. M. Whitesides, J. Am. Chem. Soc. 121, 7895 (1999).

${ }^{5}$ Y. A. Ovchenkov, H. Geisler, J. M. Burst, S. N. Thornburg, C. A. Ventrice Jr., Chunjuan Zhang, J. Redepenning, Y. Losovyj, Luis Rosa, P. A. Dowben, and B. Doudin, Chem. Phys. Lett. 381, 7 (2003).

${ }^{6}$ Y. Tai, A. Shaporenko, W. Eck, M. Grunze, and M. Zharnikov, Appl. Phys. Lett. 85, 6257 (2004).

${ }^{7}$ Y. Tai, A. Shaporenko, H. Noda, M. Grunze, and M. Zharnikov, Adv. Mater. (Weinheim, Ger.) 17, 1745 (2005).

${ }^{8}$ Y. Tai, A. Shaporenko, M. Grunze, and M. Zharnikov, J. Phys. Chem. B 109, 19411 (2005).

${ }^{9}$ H. Noda, Y. Tai, A. Shaporenko, M. Grunze, and M. Zharnikov, J. Phys. Chem. B 109, 22371 (2005).
${ }^{10}$ M. Wirde, U. Gelius, T. Dunbar, and D. L. Allara, Nucl. Instrum. Methods Phys. Res. B 131, 245 (1997).

${ }^{11}$ B. Jäger, H. Schürmann, H. U. Müller, H.-J. Himmel, M. Neumann, M. Grunze, and Ch. Wöll, Z. Phys. Chem. (Munich) 202, 263 (1997).

${ }^{12}$ P. Feulner, T. Niedermayer, K. Eberle, R. Schneider, D. Menzel, A. Baumer, E. Schmich, A. Shaporenko, Y. Tai, and M. Zharnikov, Phys. Rev. Lett. 93, 178302 (2004).

${ }^{13}$ M. Zharnikov, and M. Grunze, J. Vac. Sci. Technol. B 20, 1793 (2002), and the references therein.

${ }^{14}$ W. Geyer, V. Stadler, W. Eck, M. Zharnikov, A. Gölzhäuser, and M. Grunze, Appl. Phys. Lett. 75, 2401 (1999).

${ }^{15}$ W. Eck, V. Stadler, W. Geyer, M. Zharnikov, A. Gölzhäuser, and M. Grunze, Adv. Mater. (Weinheim, Ger.) 12, 805 (2000).

${ }^{16}$ A. Gölzhäuser, W. Eck, W. Geyer, V. Stadler, T. Weimann, P. Hinze, and M. Grunze, Adv. Mater. (Weinheim, Ger.) 13, 806 (2001).

${ }^{17}$ S. Frey, H.-T. Rong, K. Heister, Y.-J. Yang, M. Buck, and M. Zharnikov, Langmuir 18, 3142 (2002).

${ }^{18}$ Y. Tai, A. Shaporenko, W. Eck, M. Grunze, and M. Zharnikov, Langmuir 20, 7166 (2004).

${ }^{19}$ D.-Q. Feng, Ya.B. Losovyj, Y. Tai, M. Zharnikov, and P. A. Dowben, , J. Mater. Chem., doi:10.1039/b609217a (2006).

${ }^{20}$ E. W. Plummer, and W. Eberhardt, Adv. Chem. Phys. 49, 533 (1982).

${ }^{21}$ H.-P. Steinrück, Vacuum 45, 715 (1994); J. Phys.: Condens. Matter 8, 6465 (1996).

${ }^{22}$ N. V. Richardson, and A. M. Bradshaw, Electron Spectroscopy: Theory, Techniques and Applications, Edited by C. R. Brundle, and A. D. Baker, Vol. 4 (Academic Press, New York, 1984), p. 153.

${ }^{23}$ P. A. Dowben, Z. Phys. Chem. 202, 227 (1997).

${ }^{24}$ P. A. Dowben, B. Xu, J. Choi, and E. Morikawa, "Band Structure and orientation of molecules adsorbates on surfaces by angle resolved electron spectroscopies" in: Handbook of Thin Film Materials, Vol. 2 Characterization and Spectroscopy of thin Films, 61-113 (2002).

${ }^{25}$ W. R. Salaneck, S. Stafström, and J.-L. Brédas, Conjugated Polymer Surfaces and Interfaces: Electronic and Chemical Structure of Interfaces for Polymer Light Emitting Devices (Cambridge University Press, Cambridge, 1996).

${ }^{26}$ A. N. Caruso, R. Rajesekaran, G. Gallup, J. Redepenning, and P. 
A. Dowben, J. Phys.: Condens. Matter 16, 845 (2004).

${ }^{27}$ S. Hasegawa, T. Mori, K. Imaeda, S. Tanaka, Y. Yashita, H. Inokuchi, H. Fijimoto, K. Seki, and N. Ueno, J. Chem. Phys. 100, 6969 (1994).

${ }^{28}$ C. Mariani, F. Allegretti, V. Corradini, G. Contini, V. Di Castro, C. Baldacchini, and M. G. Berri, Phys. Rev. B 66, 115407 (2002).

${ }^{29}$ Jiandi Zhang, D. N. Mcllroy, P. A. Dowben, Hong Zeng, G. Vidali, D. Heskett, and M. Onellion, J. Phys.: Condens. Matter 7, 7185-7194 (1995).

${ }^{30}$ G. N. Gavrila, H. Mendez, T. U. Kampen, D. R. T. Zahn, D. V. Vyalikh, and W. Braun, Appl. Phys. Lett. 85, 4657 (2004).

${ }^{31}$ N. Koch, A. Vollmer, I. Salzmann, B. Nickel, H. Weiss, and J. P. Rabe, Phys. Rev. Lett. 96, 156803 (2006).

${ }^{32}$ X. Crispin, A. Cornil, R. Friedlein, K. K. Okudaira, V. Lemaur, A. Crispin, G. Kestemont, M. Lehmann, M. Fahlman, R. Lazzaroni, Y. Geerts, G. Wendin, N. Ueno, J. L. Bredas, and W. R. Salaneck, J. Am. Chem. Soc. 126, 11889 (2004).

${ }^{33}$ J. S. Huang, and M. Kertesz, J. Phys. Chem. B 109, 12891 (2005).

${ }^{34}$ H. Yamane, S. Kera, K. K. Okudaira, D. Yoshimura, K. Seki, and N. Ueno, Phys. Rev. B 68, 033102 (2003).

${ }^{35}$ W. K. Ford, C. B. Duke, and A. Paton, J. Chem. Phys. 78, 4734 (1983); D. Yoshimura, H. Ishii, Y. Ouchi, T. Miyamae, S. Hasegawa, K. K. Okudaira, N. Ueno, and K. Seki, ibid. 120, 1075310762 (2004); E. Zojer, M. Knupfer, Z. Shuai, J. L. Bredas, J. Fink, and G. Leising, J. Phys.: Condens. Matter 12, 1753-1768 (2000).

${ }^{36}$ S. Narioka, H. Ishii, K. Edamatsu, K. Kamiya, S. Hasegawa, T. Ohta, N. Ueno, and K. Seki, Phys. Rev. B 52, 2362 (1995); H. Ishii, S. Narioka, K. Edamatsu, K. Kamiya, S. Hasegawa, T. Ohta, N. Ueno, and K. Seki, J. Electron Spectrosc. Relat. Phenom. 78, 395 (1996); H. Ishii, S. Narioka, K. Edamatsu, K. Kamiya, S. Hasegawa, T. Ohta, N. Ueno, and K. Seki, ibid. 76, 553 (1995); H. Schurmann, N. Koch, A. Vollmer, S. Schrader, and M. Neumann, Synth. Met. 111, 591 (2000).

${ }^{37}$ V. Perebeinos, and M. Newton, Chem. Phys. 319, 159-166 (2005)

${ }^{38}$ Y. Tai, A. Shaporenko, H.-T. Rong, M. Buck, W. Eck, M. Grunze, and M. Zharnikov, J. Phys. Chem. B 108, 16806 (2004).

${ }^{39}$ W. Azzam, B. I. Wehner, R. A. Fischer, A. Terfort, and C. Wöll, Langmuir 18, 7766 (2002).

${ }^{40}$ A. Ulman, Chem. Rev. (Washington, D.C.) 96, 1533 (1996); edited by A. Ulman, Thin films: self-assembled monolayers of thiols (Academic Press: San Diego, CA, 1998); J. C. Love, L. A. Estroff, J. K. Kriebel, R. G. Nuzzo, and G. M. Whitesides, Chem. Rev. (Washington, D.C.) 105, 1103 (2005).

${ }^{41}$ L. G. Rosa, Y. B. Losovyj, J. Choi, and P. A. Dowben, J. Phys. Chem. B 109, 7817 (2005).

${ }^{42}$ D. N. McIlroy, C. Waldfried, T. McAvoy, J. Choi, P. A. Dowben, and D. Heskett, Chem. Phys. Lett. 264, 168 (1997).

${ }^{43}$ D.-Q. Feng, A. N. Caruso, D. Schulz, Ya. B. Losovyj, and P. A. Dowben, J. Phys. Chem. B 109, 16382 (2005).

${ }^{44}$ P. A. Dowben, D. LaGraffe, and M. Onellion, J. Phys.: Condens. Matter 1, 6571 (1989).

${ }^{45}$ W. Eck, A. Küller, M. Grunze, B. Völkel, and A. Gölzhäuser, Adv. Mater. (Weinheim, Ger.) 17, 2583 (2005).

${ }^{46}$ E. Morikawa, J. Choi, H. M. Manohara, H. Ishii, K. Seki, K. K. Okudaira, and N. Ueno, J. Appl. Phys. 87, 4010 (2000).

${ }^{47}$ J. Choi, H. M. Manohara, E. Morikawa, P. T. Sprunger, P. A.
Dowben, and S. P. Palto, Appl. Phys. Lett. 76, 381 (2000).

${ }^{48}$ H. M. Manohara, E. Morikawa, J. Choi, and P. T. Sprunger, J. Microelectromech. Syst. 8, 417 (1999).

${ }^{49}$ J. J. P. Stewart, J. Comput. Chem. 10, 209 (1989); J. J. P. Stewart, ibid. 10, 221 (1989).

${ }^{50}$ A. J. Stone, Mol. Phys. 41, 1339 (1980).

${ }^{51}$ D. K. Chambers, S. Karanam, D. Qi, S. Selmic, Y. B. Losovyj, L. G. Rosa, and P. A. Dowben, Appl. Phys. A 80, 483 (2005).

${ }^{52}$ A. N. Caruso, D.-Q. Feng, Ya. B. Losovyj, D. Shulz, S. Balaz, L. G. Rosa, A. Sokolov, B. Doudin, and P. A. Dowben, J. Phys. Chem. B 243, 1321-1330 (2006).

${ }^{53}$ A. N. Caruso, R. Rajesh, G. Gallup, J. Redepenning, and P. A. Dowben, J. Phys. Chem. B 108, 6910 (2004).

${ }^{54}$ I. G. Hill, A. Kahn, J. Cornil, D. A. dos Santos, and J. L. Brédas, Chem. Phys. Lett. 317, 444 (2000).

${ }^{55}$ A. N. Caruso, L. Bernard, Bo Xu, and P. A. Dowben, J. Phys. Chem. B 107, 9620 (2003).

${ }^{56}$ J. Choi and P. A. Dowben, Surf. Sci. 600, 2997 (2006), and the references therein.

${ }^{57}$ A. Bilic, J. R. Reimers, and N. S. Hush, Can. J. Phys. 122, 0947708 (2005).

${ }^{58}$ A. Görling, Phys. Rev. A 54, 3912 (1996).

${ }^{59}$ G. Fronzoni, M. Stener, P. Decleva, and G. De Alti, Chem. Phys. 232, 9 (1998).

${ }^{60}$ G. C. Solomon, J. R. Reimers, and N. S. Hush, J. Chem. Phys. 121, 6615 (2004).

${ }^{61}$ D. P. Chong, and Y. Takahata, Chem. Phys. Lett. 418, 286 (2006).

${ }^{62}$ A. P. Hitchcock, S. G. Urquhart, A. T. Wen, A. L. D. Kilcoyne, T. Tyliszczak, E. Rühl, N. Kosugi, J. D. Bozek, J. T. Spencer, D. N. McIlroy, and P. A. Dowben, J. Phys. Chem. B 101, 3483-3493 (1997).

${ }^{63}$ N. N. Tytyulkov, and O. E. Polansky, Z. Naturforsch. B 32, 490 (1977).

${ }^{64}$ A. D. Miller, K. J. Gaffney, S. H. Liu, P. Szymanski, S. GarrettRoe, C. M. Wong, and C. B. Harris, J. Phys. Chem. A 106, 7636 (2002); M. C. Vargas, P. Giannozzi, A. Selloni, and G. Scoles, J. Phys. Chem. B 105, 9509 (2001); R. Rousseau, V. De Renzi, R. Mazzarello, D. Marchetto, R. Biagi, S. Scandolo, and U. del Pennino, ibid. 110, 10862 (2006); F. P. Cometto, P. ParedesOlivera, V. A. Macagno, and E. M. Patrito, ibid. 109, 21737 (2005); C. Masens, M. J. Ford, and M. B. Cortie, Surf. Sci. 580, 19 (2005); L. G. Wang, E. Y. Tsymbal, and S. S. Jaswal, J. Magn. Magn. Mater. 286, 119 (2005); A. Ferretti, and R. Di Felice, Phys. Rev. B 70, 115412 (2004); J. Nara, S. Higai, Y. Morikawa, and T. Ohno, J. Chem. Phys. 120, 6705-6711 (2004); A. N. Caruso, L. G. Wang, S. S. Jaswal, E. Y. Tsymbal, and P. A. Dowben, J. Mater. Sci., doi:10.1007/s10853-00603262-7 (2006

${ }^{65}$ Luis G. Rosa, Jie Xiao, Ya. B. Losovyj, Yi Gao, I. N. Yakovkin, Xiao C. Zeng, and P. A. Dowben, J. Am. Chem. Soc. 127, 17261 (2005); Kyungwha Park, M. R. Pederson, L. L. Boyer, W. N. Mei, R. F. Sabirianov, X. C. Zeng, S. Bulusu, S. Curran, J. Dewald, E. Day, S. Adenwalla, M. Diaz, L. G. Rosa, S. Balaz, and P. A. Dowben, Phys. Rev. B 73, 035109 (2006).

${ }^{66}$ J. K. Tomfohr and O. F. Sankey, Phys. Status Solidi B 233, 59 (2002); J. Chem. Phys. 120, 1542 (2004).

${ }^{67}$ J. K. Tomfohr, and O. F. Sankey, Phys. Rev. B 65, 245105 (2002).

${ }^{68}$ S. Hong, R. Reifenberger, W. Tian, S. Datta, J. Henderson, and C. P. Kubiak, Superlattices Microstruct. 28, 289 (2000). 
${ }^{69}$ C. Fuxen, W. Azzam, R. Arnold, G. Witte, A. Terfort, and Ch. Wöll, Langmuir 17, 3689 (2001).

${ }^{70}$ P. Cyganik, M. Buck, W. Azzam, and C. Wöll, J. Phys. Chem. B 108, 4989 (2004).

${ }^{71}$ W. Azzam, P. Cyganik, G. Witte, M. Buck, and C. Wöll, Langmuir 19, 8262 (2003).

${ }^{72}$ T. Ishida, W. Mizutani, U. Akiba, K. Umemura, A. Inoue, N. Choi, M. Fujihira, and H. Tokumoto, J. Phys. Chem. B 103, 1686 (1999).
${ }^{73}$ T. Ishida, W. Mizutani, N. Choi, U. Akiba, M. Fujihira, and H. Tokumoto, J. Phys. Chem. B 104, 11680 (2000).

${ }^{74}$ R. Liu, S. H. Ke, H. U. Baranger, and W. T. Yang, J. Chem. Phys. 122, 044703 (2005).

${ }^{75}$ M. Magoga, and C. Joachim, Phys. Rev. B 59, 16011-16021 (1999).

${ }^{76}$ D.-Q. Feng, D. Wisbey, Y. Tai, Ya. B. Losovyj, M. Zharnikov, and P. A. Dowben, J. Phys. Chem. B 110, 1095 (2006).

${ }^{77}$ R. Magno, and J. G. Adler, Phys. Rev. B 15, 1744 (1977). 